\title{
À propos du principe de précaution
}

La controverse qui suit concernant le principe de précaution oppose une juriste (Laurence Boy) et un economiste (Olivier Godard). Elle trouve son origine dans un texte d'O. Godard publié dans NSS en 1998 ("Le principe de précaution : renégocier les conditions de l'agir en univers controversé ", NSS 6 (1), 41-45). L. Boy a déjà contesté l'interprétation juridique du principe de précaution avancée par O. Godard dans un article publié par NSS en 1999 ( La nature juridique du principe de précaution ", NSS 7 (3), 5-11). O. Godard a répondu à ces critiques dans le numéro 2 (Avril-Juin 2000) de NSS ("Le principe de précaution, règle impérative ou principe possibiliste en appelant au jugement ", NSS 8 (2), 56-57). Ci-dessous L. Boy maintient et développe son analyse de juriste et $O$. Godard explique pourquoi les nouveaux arguments qu'elle met en avant ne remettent pas en cause sa propre analyse.

Le principe de précaution s'avérant de plus en plus comme une référence majeure dans la conduite des affaires aussi bien privées que publiques, une controverse portant sur son statut et sa portée à la fois politiques et juridiques est évidemment d'un intérêt primordial. II est important de pouvoir définir avec précision l'outil qu'il constitue tant pour le juge que pour le législateur. D'où le caractère qui pourra apparaître très technique des arguments que s'opposent $L$. Boy et $O$. Godard. Que l'arbre ne cache pas la forêt ! Derrière ces arguments, ce sont deux conceptions du principe de précaution qui s'affrontent et c'est la question de savoir ce qu'il adviendra de lui, en particulier, à travers la possibilité ou non pour le juge de s'appuyer sur lui pour contraindre les comportements des acteurs privés, qui est posée.

L. Boy puise dans la notion, plutôt flexible, de "standard * juridique, vue comme un des instruments par lesquels le droit s'incorpore des matières nouvelles, des arguments pour ouvrir au juge des possibilités allant dans ce sens. Plus, elle voit dans cette ouverture la condition même pour que le principe de précaution acquière une réelle force juridique - et, donc, pratique. $O$. Godard au contraire privilégie une approche politique de la précaution centrée sur le débat public, voyant dans l'intervention du juge une régression plus qu'une avancée ; et cela le conduit à une analyse affinée - et volontiers restrictive - des textes pris "à la lettre". Ainsi se dessine sans doute l'enjeu majeur qui existe aujourd'hui - à ce moment clé où il prend corps - autour du principe de précaution. L'intérêt de cette controverse est de mettre en évidence les termes de ce débat. Et, à partir de là, d'inviter à deux tâches.

Elle invite en premier lieu à continuer à s'interroger sur les fondements mêmes - et le bienfondé - de l'alternative sur laquelle il repose. L. Boy y voit l'effet d'une connaissance imparfaite du droit par le non juriste ( $f$. son article cité ci-dessus, p. 6). Pour elle, il s'agit en somme d'un pur et simple artefact de l'interdisciplinarité ; et, qui plus est, d'un artefact fâcheux puisqu'il est susceptible de conséquences négatives quant à l'efficacité juridique du principe de précaution. C'est évidemment là une affirmation qui mérite tout particulierement de retenir l'attention dans une réflexion sur l'interdisciplinarité. Mais peut-on s'en tenir à cette explication? Cela n'apparaît en effet pas aussi simple, car les auteurs font eux-mêmes état de positions divergentes sur la question chez les juristes (C'est ainsi que L. Boy reproche à $O$. Godard de s'aligner sur celle de F. Ewald, autre juriste auquel elle oppose sa propre analyse). Cela dit, on aimerait en effet savoir pourquoi l'économiste éprouve le besoin de se frotter de façon aussi fouillée au droit, comment il le voit et la place qu'il lui donne dans sa démarche, ce qui permettrait sans doute de comprendre pourquoi il fait sienne telle approche juridique du principe de précaution plutôt que telle autre.

Elle invite par ailleurs à suivre les évolutions des applications du principe de précaution et elle offre une grille d'analyse permettant de voir dans quel sens elles vont par rapport aux deux branches de cette alternative. Après tout, n'est-ce pas " sur le terrain * que le débat - posé ici en termes que l'on pourrait qualifier de normatifs - se tranchera ? II restera alors à comprendre ce qui commande ces évolutions et à mettre en évidence les conséquences qui en découlent. 